\title{
Immune Microenvironment and Clinical Significance of PD1 Expression in Hepatocellular Carcinoma Patients
}

\section{Lili Yang}

First Affiliated Hospital Zhejiang University

\section{Jili Wang}

First Affiliated Hospital Zhejiang University

\section{Linpeng Yao}

First Affiliated Hospital Zhejiang University

Cheng Chen

Zhejiang Chinese Medical University

\section{Junhan Pan}

First Affiliated Hospital Zhejiang University

\section{Ling Peng}

Zhejiang Provincial People's Hospital

Feng Chen ( $\nabla$ chenfenghz@zju.edu.cn )

First Affiliated Hospital Zhejiang University

\section{Research Article}

Keywords: Programmed death 1(PD1), immune microenvironment, Hepatocellular carcinoma (HCC), liver disease, Survival

Posted Date: February 2nd, 2022

DOI: https://doi.org/10.21203/rs.3.rs-1257996/v1

License: (c) (1) This work is licensed under a Creative Commons Attribution 4.0 International License. Read Full License 


\section{Abstract}

Immune checkpoint inhibitors (ICl) have revolutionized treatment for hepatocellular carcinoma (HCC), however, durable response is limited to only a subset of patients. Therefore, this study aims to identify the ICl-responsive HCC patients and to investigate the distribution of programmed death 1 (PD1) in HCC patients, as well as the relationship among PD1, pathological parameters and survival of HCC patients. A total of 372 HCC patients (western population) from The Cancer Genome Atlas (TCGA), 115 Primary HCC tissue and 52 para-carcinoma tissue (Data Set GSE76427, eastern population) from Gene Expression Omnibus (GEO) database were enrolled. Bioinformatics analysis were conducted with R software and Tumor Immune Estimation Resource (TIMER). This study showed the expression of PD1 was upregulated in both western and eastern HCC patients, which was positively correlated with patients' BMI and AFP value, as well as prognosis. The expression level of PD1 was significantly correlated with immune cell infiltration. Our study reveals the relationship of PD-1 and the HCC tumor microenvironment, providing insight for new ICl therapeutic options. Besides, PD1 expression levels in hepatocellular carcinoma (HCC) patients may be a survival predictor.

\section{Introduction}

Hepatocellular carcinoma (HCC) is one of the most common malignant tumors in digestive tract, it is characterized by insidious onset, rapid progression, high malignancy degree and poor prognosis[1, 2]. It ranks seventh in incidence and second in mortality among all tumors [3, 4]. HCC is extremely prevalent in Asia, and its incidence and death accounts for more than $50 \%$ worldwide, which poses a serious threat to the health of Chinese residents and a heavy burden on the social economy[4-6]. Currently, surgery is the main treatment for HCC[7]. However, for patients in the middle and advanced stages or those who lose the chance of surgery due to other reasons, only local treatment such as transhepatic arterial chemo therapy and embolization, percutaneous ethanol injection, radiofrequency ablation, radiotherapy, etc. can be adopted, whose efficacy was very limited[3]. With the development of science, immunotherapy has gradually become a new method of tumor treatment, and has achieved remarkable curative effect in cancers like malignant melanoma and lung cancer $[8,9]$. In recent years, clinical trials indicating that Programmed cell Death receptor 1 (PD1) inhibitors could effectively improve the survival of patients with advanced HCC have also been reported[10-12]. For instance, El-Khoueiry et al. reported that nivolumab, a PD1 inhibitor, had a certain effect on advanced HCC in 2017[10]. In the next year, Zhu et al. reported that Pembrolizumab, another PD1 inhibitor, could be used as a second-line therapy for advanced HCC[11]. Although immunotherapy was effective in patients with HCC, only $10-20 \%$ of patients had significant benefit[13].Therefore, how to identify patients who have a positive response to PD1 inhibitors becomes a critical issue $\square$ And based on this issue, analyzing the distribution of PD1 in HCC and its relationship with clinicopathological parameters and prognosis of HCC patients has become a primary task.

In this research, We conducted a preliminary ibionformatics analysis on PD1 expression along with its correlation to clinicopathological parameters, and we also compared the prognosis between HCC patients with high PD1 expression and low PD1 expression through bioinformatics analysis, thereby providing 
certain reference for further investigation into the role of PD1 in HCC. The study protocol is performed in accordance with the relevant guidelines.

\section{Methods}

\subsection{Data sources}

The Cancer Genome Atlas(TCGA) program was launched by the National Cancer Institute(NCI) and the National Human Genome Research Institute(NHGRI) in 2006. We downloaded the data of HCC patients from UCSC Xena(https://xenabrowser.net/datapages/). After excluding the cases without survival information, datasets containing a total of $372 \mathrm{HCC}$ patients were obtained. To further explore the regional differences, we selected a dataset of easterners in Gene Expression Omnibus (GEO) database GSE76427】which was uploaded by the Bioinformatics Institute of Singapore in 2017 containing whole-genome sequencing results of 115 primary HCC tissues and 52 paracancer tissues[14].

\subsection{Data analysis}

\subsection{1 analysis of PD1 expression}

In the data from TCGA, the expression of PD1 was presented as standardized FPKM value, While in the data from GSE76427, the expression of PD1 was presented as the mRNA expression level uploaded by the author. The data was divided into cancer group(CG) and para-cancer group(PC), and a two-tailed independent sample t test statistic was used to compare difference of PD1 expression between the two groups in prism-GraphPad. The statistical significance was set at $p<0.05$.

\subsubsection{The correlation analysis of the PD1 expression and clinicopathological parameters}

The data from TCGA was divided into low expression group and high expression group according to the median of PD1 expression. Chi-square test was used to compare differences in variables of the two groups such as age, gender, race, tumor stage, tumor grade, AFP, albumin, etc. Among the above variables, AFP, albumin and other variables were also divided into low expression group and high expression group according to the median respectively. The statistical significance was set at $p<0.05$.

\subsubsection{The correlation between PD1 expression and prognosis}

The prognosis of HCC from TCGA dataset was analyzed on the online database KM Plot (https://kmplot.com/)[15]. We confirmed the optimal cut-off point of PD1 expression in HCC by KM plot database, by which the HCC patients were divided into high PD1 expression group and low PD1 expression group. Kaplan-meier (KM) survival analysis was used to analyze the survival of patients in different groups. Log-rank test was used to compare prognosis difference between the two groups. $p<0.05$ was considered statistically significant. 
X-tile software was used to confirm the optimal cut-off point of HCC data from GSE76427, by which the HCC patients were divided into high PD1 expression group and low PD1 expression group. Kaplan-meier (KM) survival analysis and log-rank test were used to analyze the prognosis difference between the two groups. The statistical analysis in this part was performed by SPSS 25.0 (SPSS Inc., Chicago, IL, USA). $\mathrm{P}<0.05$ was considered statistically significant.

\subsubsection{The relationship between PD1 expression and immune cell infiltration}

Since the expression of PD1 was closely related to immune cell infiltration, we further explored the correlation between PD1 expression and immune cell infiltration in HCC patients. The online immune infiltration database "TIMER" (https://cistrome.shinyapps.io/timer/) which was based on the TCGA was the database used in this study for assessing and evaluating the infiltration of various immune cells such as $B$ cells, $C D 4^{+} T$ cells, $C D 8^{+} T$ cells macrophages, neutrophils, dendritic cells, etc. This database established an intelligence algorithm to predict the immune cell infiltration value depending on the gene expression information, which has been proved to be highly accurate by experiments[16]. In this study, we analyzed the relationship between PD1 expression and immune cell infiltration in the HCC through TIMER database.

\section{Results}

\subsection{PD1 expression was upregulated in hepatocellular carcinoma tissue}

As shown in Figure 1, PD1 expression was significantly higher in the hepatocellular carcinoma tissue $(0.7494 \pm 0.04740)$ than that in paraneoplastic tissue $(0.3411 \pm 0.03968)$ in the 372 hepatocellular carcinoma patients from TCGA ( $p=0.0017)$. PD1 expression was also significantly higher in the hepatocellular carcinoma tissues $(104.8 \pm 2.634)$ than that in the paraneoplastic tissues $(96.07 \pm 1.880)$ in the 115 hepatocellular carcinoma patients from GSE76427 ( $p=0.0353$ ). In addition, as the expression data from TCGA were standardized, the expression values were significantly lower than those from GSE76427, and we did not further process the expression data from GSE76427 to maintain the data originality.

\subsection{PD1 expression was positively correlated with BMI and AFP}

By analyzing the correlation between PD1 expression and clinical parameters in the data from TCGA, we found that patients in the high PD1 expression group had a higher body mass index (BMI) $(p=0.041)$ and higher serum alpha-fetoprotein (AFP) $(p<0.001)$ compared to the low PD1 expression group. Only $3 \%$ of patients in the high PD1 expression group were relatively thin and $9.6 \%$ of patients in the low PD1 expression group were relatively thin, which was three times as large as the high expression group. In 
addition, among the patients in the high PD1 expression group, $63.0 \%$ of them had AFP above the median, while only $35.7 \%$ of the patients in the low PD1 expression group exceeded the median, seen in Table 1. 
Table 1

Patient characteristics by PD1 expression (From TCGA n=372).

\begin{tabular}{|c|c|c|c|c|}
\hline Characteristics & Level & Low PD1 & High PD1 & $P$ value \\
\hline \multirow[t]{2}{*}{ Age at diagnosis (yo) } & $\leq 60$ & $84(45.2 \%)$ & $93(50.0 \%)$ & 0.350 \\
\hline & $>60$ & $102(54.8 \%)$ & $93(50.0 \%)$ & \\
\hline \multirow[t]{2}{*}{ Sex } & Female & $58(31.2 \%)$ & 63(33.9\%) & 0.580 \\
\hline & Male & $128(68.8 \%)$ & $123(66.1 \%)$ & \\
\hline \multirow[t]{4}{*}{ race } & White & $102(54.8 \%)$ & $83(44.6 \%)$ & 0.180 \\
\hline & Black & $8(4.3 \%)$ & $10(5.4 \%)$ & \\
\hline & Asian & $73(39.2 \%)$ & $86(46.2 \%)$ & \\
\hline & Unknown & $3(1.6 \%)$ & $7(3.8 \%)$ & \\
\hline \multirow[t]{3}{*}{ BMI } & $<18.5$ & $16(9.6 \%)$ & $5(3.0 \%)$ & 0.041 \\
\hline & $18.5 \sim 24.9$ & $74(44.6 \%)$ & $82(48.5 \%)$ & \\
\hline & $\geq 25$ & $76(45.8 \%)$ & $82(48.5 \%)$ & \\
\hline \multirow[t]{3}{*}{ Family cancer history } & Yes & 63(33.9\%) & $49(26.3 \%)$ & 0.281 \\
\hline & No & $99(53.2 \%)$ & $109(58.6 \%)$ & \\
\hline & Unknown & $24(12.9 \%)$ & $28(15.1 \%)$ & \\
\hline \multirow[t]{5}{*}{ Grade } & $\mathrm{G} 1$ & $35(18.8 \%)$ & $20(10.8 \%)$ & 0.073 \\
\hline & $\mathrm{G} 2$ & $92(49.5 \%)$ & $86(46.2 \%)$ & \\
\hline & G3 & $52(28.0 \%)$ & $70(37.6 \%)$ & \\
\hline & G4 & $4(2.2 \%)$ & $8(4.3 \%)$ & \\
\hline & Unknown & $3(1.6 \%)$ & $2(1.1 \%)$ & \\
\hline \multirow[t]{5}{*}{ Stage } & 1 & $85(45.7 \%)$ & $87(46.8 \%)$ & 0.597 \\
\hline & $\|$ & $42(22.6 \%)$ & $44(23.7 \%)$ & \\
\hline & III & $41(22.0 \%)$ & $44(23.7 \%)$ & \\
\hline & IV & $4(2.2 \%)$ & $1(0.5 \%)$ & \\
\hline & Unknown & $14(7.5 \%)$ & $10(5.4 \%)$ & \\
\hline \multirow[t]{3}{*}{ Child-Pugh grade } & A & $121(65.1 \%)$ & $97(52.2 \%)$ & 0.045 \\
\hline & B & $11(5.9 \%)$ & $10(5.4 \%)$ & \\
\hline & C & $0(0.0 \%)$ & $1(0.5 \%)$ & \\
\hline
\end{tabular}




\begin{tabular}{|c|c|c|c|c|}
\hline Characteristics & Level & Low PD1 & High PD1 & $P$ value \\
\hline & Unknown & $54(29.0 \%)$ & $78(41.9 \%)$ & \\
\hline \multirow[t]{4}{*}{ Vascular invasion } & Macro & $7(3.8 \%)$ & $9(4.8 \%)$ & 0.945 \\
\hline & Micro & $47(25.3 \%)$ & $46(24.7 \%)$ & \\
\hline & None & $105(56.5 \%)$ & $102(54.8 \%)$ & \\
\hline & Unknown & $27(14.5 \%)$ & $29(15.6 \%)$ & \\
\hline \multirow[t]{2}{*}{ Albumin } & Low & $81(51.6 \%)$ & $77(54.6 \%)$ & 0.602 \\
\hline & High & $76(48.4 \%)$ & $64(45.4 \%)$ & \\
\hline \multirow[t]{2}{*}{ Creatinine } & Low & $84(52.8 \%)$ & $80(56.7 \%)$ & 0.497 \\
\hline & High & $75(47.2 \%)$ & $61(43.3 \%)$ & \\
\hline \multirow[t]{2}{*}{ AFP } & Low & $90(64.3 \%)$ & $51(37.0 \%)$ & $<0.001$ \\
\hline & High & $50(35.7 \%)$ & $87(63.0 \%)$ & \\
\hline \multirow[t]{2}{*}{ platelet } & Low & $87(53.7 \%)$ & $78(54.5 \%)$ & 0.883 \\
\hline & High & $75(46.3 \%)$ & $65(45.5 \%)$ & \\
\hline \multirow[t]{2}{*}{ prothrombin } & Low & $92(58.6 \%)$ & $88(63.8 \%)$ & \\
\hline & High & $65(41.4 \%)$ & $50(36.2 \%)$ & \\
\hline \multirow[t]{6}{*}{ surgery } & Segmentectomy, multiple & $44(23.7 \%)$ & $43(23.1 \%)$ & 0.348 \\
\hline & Segmentectomy, single & $40(21.5 \%)$ & $49(26.3 \%)$ & \\
\hline & Lobectomy & $77(41.4 \%)$ & $64(34.4 \%)$ & \\
\hline & Extended lobectomy & $14(7.5 \%)$ & $11(5.9 \%)$ & \\
\hline & Total Hepatectomy with Transplant & $0(0.0 \%)$ & $1(0.5 \%)$ & \\
\hline & Unknown & $2(1.1 \%)$ & $1(0.5 \%)$ & \\
\hline \multirow[t]{3}{*}{ Radiation } & Yes & $4(2.2 \%)$ & $3(1.6 \%)$ & 0.892 \\
\hline & No & $145(78.0 \%)$ & $148(79.6 \%)$ & \\
\hline & Unknown & $37(19.9 \%)$ & $35(18.8 \%)$ & \\
\hline Post operation & Yes & $16(8.6 \%)$ & $9(4.8 \%)$ & 0.294 \\
\hline \multirow[t]{2}{*}{ ablation embolization } & No & $131(70.4 \%)$ & $141(75.8 \%)$ & \\
\hline & Unknown & $39(21.0 \%)$ & $36(19.4 \%)$ & \\
\hline Prior malignancy & Yes & $165(88.7 \%)$ & $172(92.5 \%)$ & 0.214 \\
\hline
\end{tabular}


Level

No
Low PD1

21(11.3\%)
High PD1

$14(7.5 \%)$

Additionally, it is interesting to note that the tumor grades in high PD1 expression group were more concentrated in the G3, G4 stage (41.9\%), whereas only $30.2 \%$ of the tumors in the low PD1 expression group were in high grade. Although there was no statistical difference $(p=0.073)$, the trend of higher tumor grade in the high PD1 expression group needs to be taken into account. The remaining clinical parameters such as age, gender, race, vascular infiltration and stage did not showed significant correlation with PD1 expression. The data from Singapore showed similar results (Table 2).

Table 2

Patient characteristics by PD1 expression (GSE76427).

\begin{tabular}{|c|c|c|c|c|}
\hline Characteristics & Level & Low PD1 $(n=29)$ & $\begin{array}{l}\text { High PD1 } \\
(n=86)\end{array}$ & $P$ value \\
\hline \multirow[t]{2}{*}{ Age at diagnosis } & $\leq 60$ & $8(27.6 \%)$ & $40(46.5 \%)$ & 0.117 \\
\hline & $>60$ & $21(72.4 \%)$ & $46(53.5 \%)$ & \\
\hline \multirow[t]{2}{*}{ Sex } & Male & $25(86.2 \%)$ & $68(79.1 \%)$ & 0.567 \\
\hline & Female & $4(13.8 \%)$ & $18(20.9 \%)$ & \\
\hline \multirow[t]{4}{*}{ BCLC stage } & A & $19(65.5 \%)$ & $55(64.0 \%)$ & 0.468 \\
\hline & B & $8(27.6 \%)$ & $20(23.3 \%)$ & \\
\hline & C & $2(6.9 \%)$ & $7(8.1 \%)$ & \\
\hline & NA & $0(0.0 \%)$ & $4(4.7 \%)$ & \\
\hline \multirow[t]{5}{*}{ TNM stage } & I & $14(48.3 \%)$ & $41(47.7 \%)$ & 0.940 \\
\hline & II & $9(31.0 \%)$ & $26(30.2 \%)$ & \\
\hline & III & $5(17.2 \%)$ & $16(17.6 \%)$ & \\
\hline & IV & $1(3.4 \%)$ & $2(2.4 \%)$ & \\
\hline & NA & $0(0.0 \%)$ & $1(1.2 \%)$ & \\
\hline \multirow[t]{2}{*}{ Overall survival } & Alive & $19(65.5 \%)$ & 73(84.9\%) & 0.047 \\
\hline & Dead & $10(34.5 \%)$ & $13(15.1 \%)$ & \\
\hline \multirow[t]{3}{*}{ Relapse free survival } & No relapse & $13(44.8 \%)$ & $47(54.7 \%)$ & 0.465 \\
\hline & Relapse & $13(44.8 \%)$ & $35(40.7 \%)$ & \\
\hline & NA & $3(10.3 \%)$ & $4(4.7 \%)$ & \\
\hline
\end{tabular}




\subsection{Better prognosis in patients with high PD1 expression}

The prognosis of hepatocellular carcinoma patients with different PD1 expression was shown in Figure 2. We found that the overall survival and the disease-free survival of patients in the high PD1 expression group from TCGA were significantly higher than those in the low expression group as were shown in KM survival curve and log-rank test (Figure 2A and Figure 2B). The median overall survival of the high PD1 expression group was 70.5 months and the recurrence-free survival was 37.67 months. The median overall survival of the low PD1 expression group was 37.8 months and the recurrence-free survival was 18.87 months, which was significantly lower than that of the high expression group (all $p<0.05$ ).

In the GSE76427 dataset from Singapore, We also confirmed that the high PD1 express group showed improved survival than that in the low PD1 expression group (Figure $2 C, p<0.05$ ). The median overall survival in the high PD1 expression group was 6.29 years (75.4 months), and 3.96 years (47.52 months) in the low PD1 expression group.

115 HCC samples from GSE76427 dataset was included to evaluate the independent performance of the prognostic score in prognosis prediction. A nomogram based on these five pretreatment clinical variables (including age, sex, BCLC stage, TNM stage and PD1 expression) was developed to quantify survival probability in HCC patients at 1 and 3 years (Fig. 3A). The calibration curves were well matched with the idealized $45^{\circ}$ line (Fig. 3B-C), and the C-index was 0.748 , indicating this model predicts well for actual results.

\subsection{PD1 expression was significantly correlated with the degree of immune cell infiltration}

The PD1 expression level and the degree of immune cell infiltration were shown in Figure 4. As is showed in the figure, PD1 expression was positively correlated with infiltrations of $B$ cell $(r=0.511, p<0.001)$, CD8+ T cells $(r=0.544, p<0.001)$, CD4+ T cells $(r=0.272, p<0.001)$, macrophages $(r=0.316, p<0.001)$, neutrophils $(r=0.27, p<0.001)$, dendritic cell infiltration $(r=0.495, p<0.001)$.

\section{Discussion}

HCC is a serious health threat around the world as it high mobidity and mortality. As its insidious onset and rapid progression, HCC is usually diagnosed at an advanced stage, which is not suitable for surgical treatment. The molecular mechanisms in pathological process of HCC are extremely complex and researches on proteins related to HCC will help us understand the microscopic oncogenic mechanisms.

PD1 is a member of the immunoglobulin superfamily, which was mainly expressed in immune cells such as T cells, B cells and macrophages[17]. In previous research, it was found that the expression of PD1 was positively correlated with the patient prognosis in different kinds of tumors[18]. At the meantime many recent studies proved that the expression of PD1 in HCC was related to the survival of patients with 
advanced HCC and PD1 inhibitors could effectively improve their survival[18]. In addition, many works has shown that high PD1 expression was associated with adverse clinicopathological features and significantly correlated with the tumor invasiveness such as size, differentiation and venous thrombosis, AFP level and microvascular infiltration[19-21]. Although immunotherapy based on PD1 as target was effective in patients with HCC, only a few patients can actually benefit from it[13]. Therefore, it is critical to identify patients who have a positive response to PD1 inhibitors and analyze the distribution of PD1 in $\mathrm{HCC}$ and its relationship with clinicopathological parameters and prognosis of HCC patients. In this study, we analyzed gene sequencing data of HCC from TCGA and GEO to discover the of PD1 and analyzed the correlation between PD1 expression and prognosis, BMI as well as AFP level.

Previously, Li et al. found that the immune checkpoint protein PD1 was abundantly expressed in HCC, and the polymorphism of PD1 gene was related to the occurrence of liver cirrhosis and HCC[22, 23]. Li et al. further analyzed the distribution of PD1-posotive cells in 77 HCC specimens by immunohistochemistry, and the result showed that PD1-positive T cells were mainly distributed at the junction of HCC and normal adjacent tissues, mostly in the hepatic hilar region, and only a small number of PD1-positive T cells existed in HCC[19]. In current study, we also found that PD1 mRNA level in HCC tissues was significantly higher than that in paracancer tissues suggesting that immune cells with high expression of PD1 were clustered in $\mathrm{HCC}$, which was consistent with results of $\mathrm{Li}$ et al. As the finding of Pare et al. that the expression of PD1 was positively correlated with the patient prognosis, the case being that the higher the expression of PD1, the better the prognosis, in 33 kinds of tumors[18], our results proved that the positive correlation between PD1 expression and prognosis still existed in HCC patients. And our results also shown that the expression of PD1 was positively correlated with AFP level and tumor grade indicating that patients with high PD1 expression were likely to develop the HCC with higher invasiveness and higher malignancy degree, which was consistent with previous findings [20,21]. However, our study did not find the relationship between PD1 expression and T stage, vascular embolism orvascular infiltration, which may be caused by different sample sources and different detection methods.

And what is puzzling that patients with high PD1 expression showed better prognosis even though the tumor showed ehnahnced aggressive morphology (higher AFP, higher grade) in the patients with high expression of PD1 as is shown by our data and previous studies. There are several possible explanations for this phenomenon: (1) More immune cells clustered around the cancer foci to play a stronger killing effect. It can be seen in Figure 4 that the high expression of PD1 was significantly related to the infiltration of $\mathrm{B}$ cell, $\mathrm{CD} 8^{+} \mathrm{T}$ cell and dendritic cell etc. The aggregation of immune cells may be more effective at killing tumors, which improves the prognosis in patients with high PD1 expression. (2) Patients with high PD1 expression are more likely to benefit from conventional treatment. Previous literature has reported that $\mathrm{CD} 8^{+} \mathrm{T}$ cells with high PD1 expression are more prone to cell depletion and these patients may be more sensitive to immune checkpoint therapy and are potentially responsive groups for anti-PD1 therapy[20]. We speculated that these patients may also be more sensitive to chemotherapy, so the prognosis is better. Although HCC patients with high PD1 expression tend to have higher tumor grade, they may be potential beneficiaries of immunotargeted therapy. Therefore, PD1 
expression level may be the indicator and prognosis evaluation index of immunotargeted therapy for HCC patients. In order to verify our speculation further analysis on whether HCC patients with different PD1 expression levels have different reactions to chemotherapy and targeted therapy is necessary in the future.

In addition, we also found that the expression of PD1 was positively correlated with BMI. As many studies confirmed that overweight and obesity were related to high malignancy degree of tumor and were the risk factors of adverse treatment outcome and prognosis in malignant tumors[24, 25], our result showed that the higer BMI, the higher PD1 expression level which indicated the higher tumor aggressiveness. Although the underlying mechanism of this connection between BMI and PD1 needs further study, BMI maybe also a crucial influence factor in immunotargeted therapy to HCC targeting high PD1 expression and HCC patients with high BMI may might be beneficiaries of tumor treatment. However, further study is needed to verify the influence of BMI in HCC

In conclusion, in this study, we investigated the expression of PD1 in HCC, along with its relationship with clinicopathological parameters and prognosis by bioinformatics, and we also innovatively studied the expression of PD1 in HCC from Western and Eastern population simultaneously. We found that the expression of PD1 was significantly up-regulated in HCC and positively correlated with BMI, AFP, etc, and the HCC patients with high PD1 expression and high BMI may be indicative of a good prognosis.

Our study still has the following limitations: (1)The expression of PD1 in HCC was only preliminarily analyzed by bioinformatics analysis, and it was not verified by further experiments such as qRT-PCR and western blot. (2) The analysis of the correlation between PD1 and HCC was superficial, and the mechanism was not further analyzed. (3)The indication of good prognosis based on high PD1 expression may be influenced by confounding factors, such as potential influence from targeted therapy and other treatment methods. However, we cannot further analyze the confounding factors due to the current data level. Despite the above problems, we uncovered up-regulated expression of PD1 in HCC and revealed significant correlation between PD1 expression and the prognosis of HCC through preliminary analysis. which lays foundation for future study. It's worth noting that further experiments are needed to confirm these finding.

\section{Declarations}

\section{Ethics approval and consent to participate}

None.

\section{Consent for publication}

Not-applicable.

\section{Availability of data and materials}


The datasets used and/or analyzed are available fromUCSC Xena:

https://xenabrowser.net/datapages/;GEO repository:https://www.ncbi.nlm.nih.gov/geo/;and the online immune infiltration database "TIMER":https://cistrome.shinyapps.io/timer/.

\section{Competing interests}

The authors have no competing interests related to this article.

\section{Funding}

This manuscript was not funded.

\section{Authors' contributions}

LiliYang and JiliWang were involved in design and data interpretation. LiliYang wrote the manuscript. LinpengYao, ChengChen, JunhanPan and LingPeng contributed to its conception and to data analysis. Feng Chen conducted critical revision of the manuscript.

\section{Acknowledgements}

None.

\section{References}

1. Tohyama, T., et al., Pharyngeal metastasis following living-donor liver transplantation for hepatocellular carcinoma: a case report and literature review. World J Surg Oncol, 2020. 18(1): p. 109.

2. Cai, H., et al., High expression of TOP2A in hepatocellular carcinoma is associated with disease progression and poor prognosis. Oncol Lett, 2020. 20(5): p. 232.

3. Forner, A., M. Reig, and J. Bruix, Hepatocellular carcinoma. Lancet, 2018. 391(10127): p. 1301-1314.

4. Bray, F., et al., Global cancer statistics 2018: GLOBOCAN estimates of incidence and mortality worldwide for 36 cancers in 185 countries. CA Cancer J Clin, 2018. 68(6): p. 394-424.

5. Moon, A.M., A.G. Singal, and E.B. Tapper, Contemporary Epidemiology of Chronic Liver Disease and Cirrhosis. Clin Gastroenterol Hepatol, 2020. 18(12): p. 2650-2666.

6. Siegel, R.L., K.D. Miller, and A. Jemal, Cancer statistics, 2019. CA Cancer J Clin, 2019. 69(1): p. 7-34.

7. Leoni, S., et al., Treatment of Combined Hepatocellular and Cholangiocarcinoma. Cancers (Basel), 2020. 12(4).

8. Sanmamed, M.F. and L. Chen, A Paradigm Shift in Cancer Immunotherapy: From Enhancement to Normalization. Cell, 2018. 175(2): p. 313-326.

9. Yu, Y., et al., Association of Survival and Immune-Related Biomarkers With Immunotherapy in Patients With Non-Small Cell Lung Cancer: A Meta-analysis and Individual Patient-Level Analysis. 
JAMA Netw Open, 2019. 2(7): p. e196879.

10. El-Khoueiry, A.B., et al., Nivolumab in patients with advanced hepatocellular carcinoma (CheckMate 040): an open-label, non-comparative, phase 1/2 dose escalation and expansion trial. Lancet, 2017. 389(10088): p. 2492-2502.

11. Zhu, A.X., et al., Pembrolizumab in patients with advanced hepatocellular carcinoma previously treated with sorafenib (KEYNOTE-224): a non-randomised, open-label phase 2 trial. Lancet Oncol, 2018. 19(7): p. 940-952.

12. Deng, H., et al., Dual Vascular Endothelial Growth Factor Receptor and Fibroblast Growth Factor Receptor Inhibition Elicits Antitumor Immunity and Enhances Programmed Cell Death-1 Checkpoint Blockade in Hepatocellular Carcinoma. Liver Cancer, 2020. 9(3): p. 338-357.

13. Waidmann, O., Recent developments with immunotherapy for hepatocellular carcinoma. Expert Opin Biol Ther, 2018. 18(8): p. 905-910.

14. Grinchuk, O.V., et al., Tumor-adjacent tissue co-expression profile analysis reveals pro-oncogenic ribosomal gene signature for prognosis of resectable hepatocellular carcinoma. Mol Oncol, 2018. 12(1): p. 89-113.

15. Nagy, A., et al., Validation of miRNA prognostic power in hepatocellular carcinoma using expression data of independent datasets. Sci Rep, 2018. 8(1): p. 9227.

16. Li, B., et al., Comprehensive analyses of tumor immunity: implications for cancer immunotherapy. Genome Biol, 2016. 17(1): p. 174.

17. Stenzel, P.J., et al., Prognostic and Predictive Value of Tumor-infiltrating Leukocytes and of Immune Checkpoint Molecules PD1 and PDL1 in Clear Cell Renal Cell Carcinoma. Transl Oncol, 2020. 13(2): p. 336-345.

18. Pare, L., et al., Association between PD1 mRNA and response to anti-PD1 monotherapy across multiple cancer types. Ann Oncol, 2018. 29(10): p. 2121-2128.

19. Li, Z., et al., Expression and clinical significance of PD1 in hepatocellular carcinoma tissues detected by a novel mouse anti-human PD1 monoclonal antibody. Int J Oncol, 2018. 52(6): p. 2079-2092.

20. Kim, H.D., et al., Association Between Expression Level of PD1 by Tumor-Infiltrating CD8(+) T Cells and Features of Hepatocellular Carcinoma. Gastroenterology, 2018. 155(6): p. 1936-1950 e17.

21. Chang, H., et al., Expression and prognostic significance of programmed death protein 1 and programmed death ligand-1, and cytotoxic T lymphocyte-associated molecule-4 in hepatocellular carcinoma. APMIS, 2017. 125(8): p. 690-698.

22. Li, Z., et al., Immune checkpoint proteins PD-1 and TIM-3 are both highly expressed in liver tissues and correlate with their gene polymorphisms in patients with HBV-related hepatocellular carcinoma. Medicine (Baltimore), 2016. 95(52): p. e5749.

23. Li, Z., et al., Genetic variations of PD1 and TIM3 are differentially and interactively associated with the development of cirrhosis and HCC in patients with chronic HBV infection. Infect Genet Evol, 2013. 14: p. 240-6. 
24. Hashimoto, M., et al., Influence of higher BMI for hepatitis B- and C-related hepatocellular carcinomas. Langenbecks Arch Surg, 2017. 402(5): p. 745-755.

25. Picon-Ruiz, M., et al., Obesity and adverse breast cancer risk and outcome: Mechanistic insights and strategies for intervention. CA Cancer J Clin, 2017. 67(5): p. 378-397.

\section{Figures}
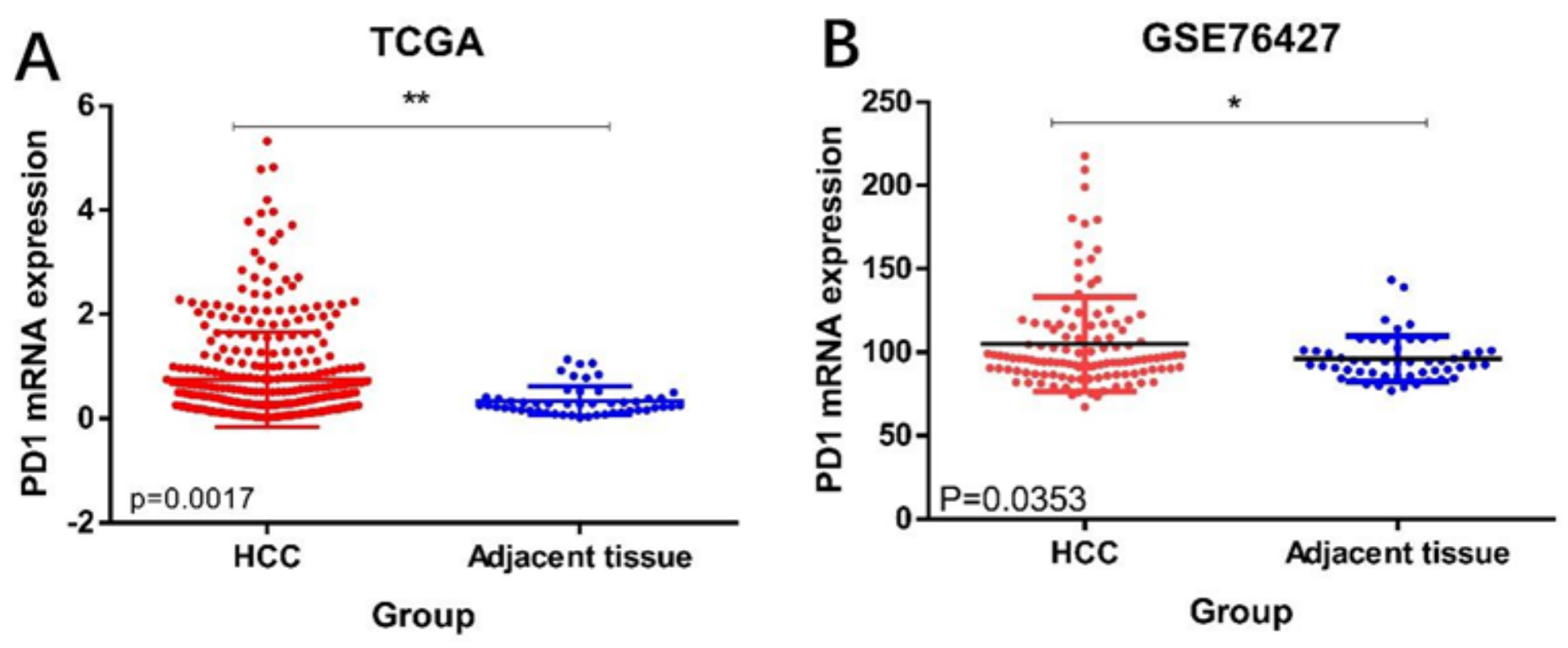

Figure 1

PD1 expression was upregulated in hepatocellular carcinoma tissue.(A) The correlation of PD1 mRNA expression between hepatocellular carcinoma tissue and Adjacenttissue in the patients from TCGA.

(B)The correlation of PD1 mRNA expression between hepatocellular carcinoma tissue and Adjacenttissue in the patients from GSE76427. 
A

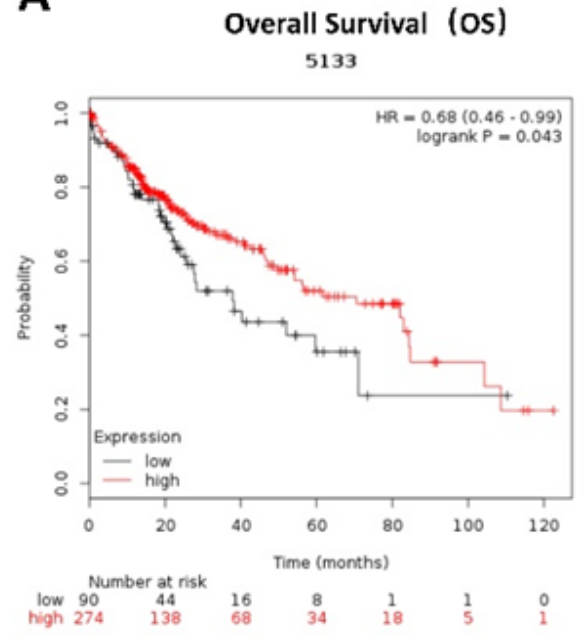

B

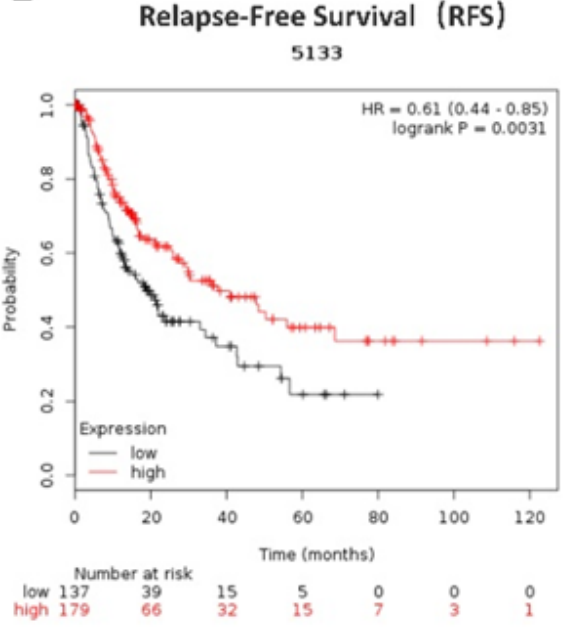

C

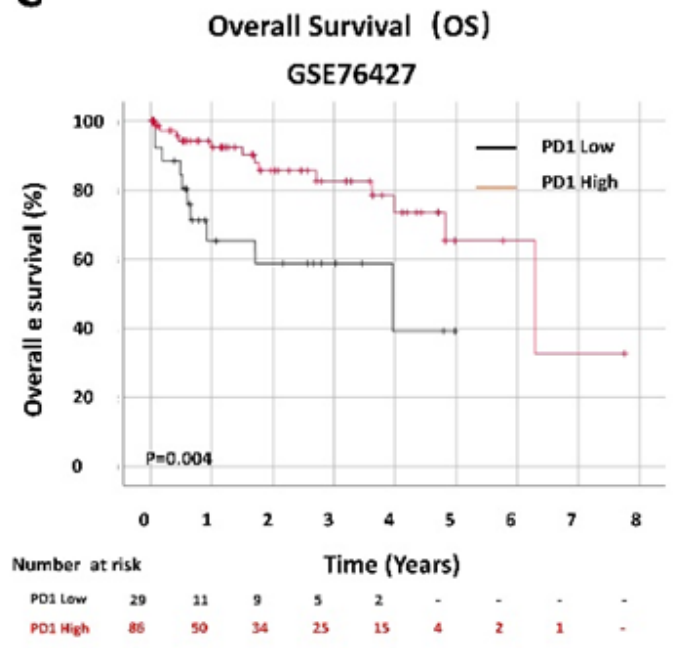

Figure 2

The prognosis of hepatocellular carcinoma patients with different PD1 expression.(A-B)Kaplan-Meier curves for the overall survival (OS) and relapse-free survival (RFS) of patients (TCGA)relative to the risk score (high or low, as defined by the median of PD1 expression). (C) Kaplan-Meier curves for the overall survival (OS) of patients (GSE76427) relative to the risk score (high or low, as defined by the median of PD1 expression). 
A

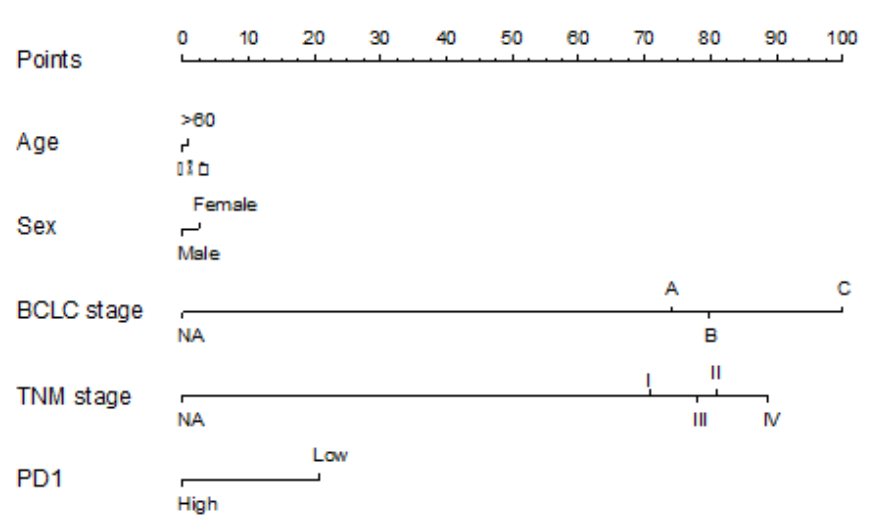

Total Points

G Year Survival

10 Year survival
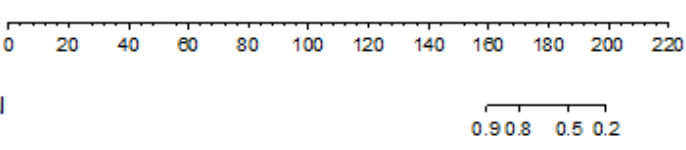

$\begin{array}{ccc}0.90 .8 & 0.5 & 0.81\end{array}$

B
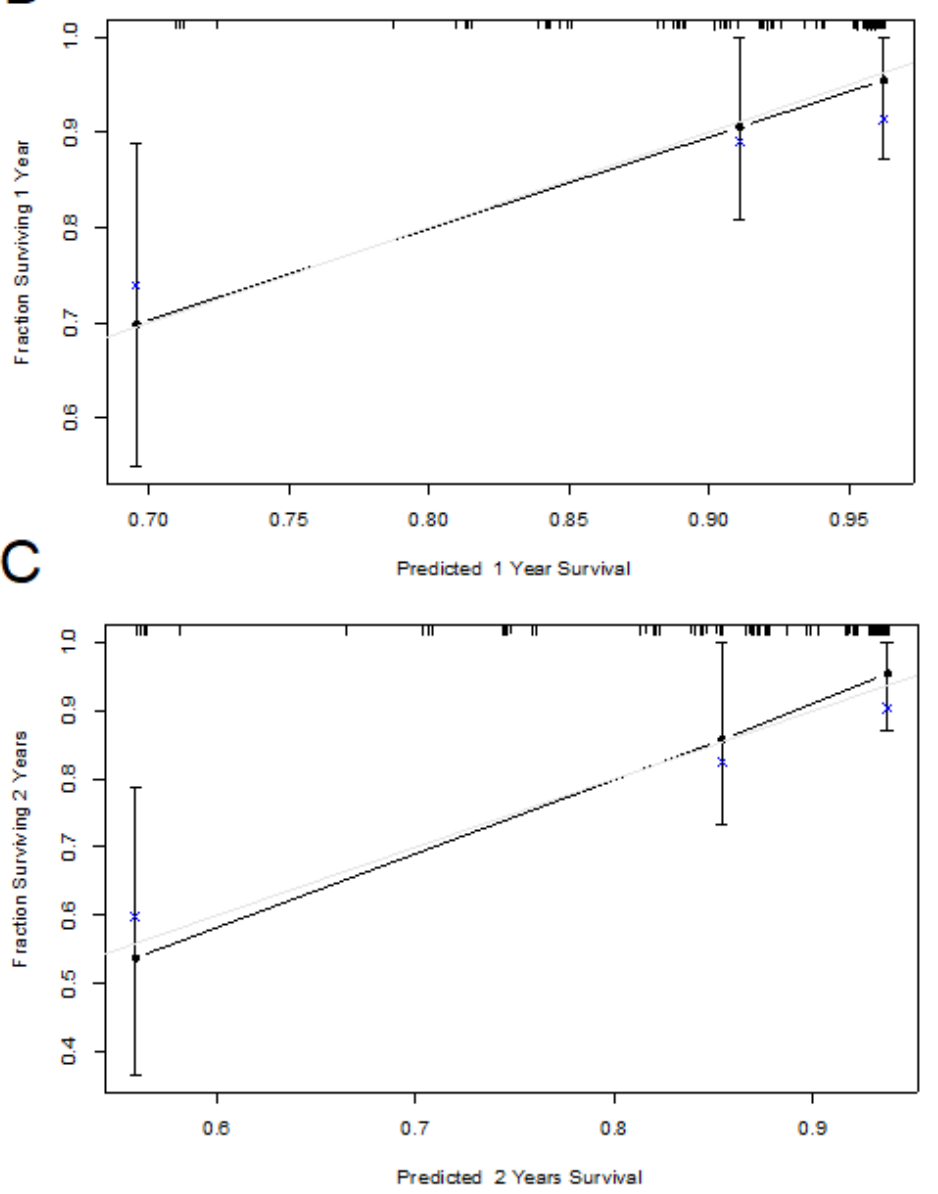

Figure 3

The independence of the prognosis modeland its correlation with clinical andpathological features insurvival prediction(GSE76427).(A)a predictive Nomogram for the survival probability in HCC patients at 1 and 3 years. (B-C) Calibration charts for validating the 1-year and 3-year survival probabilities between the nomogram prediction and the actual observation. BCLC,Barcelona Clinic Liver Cancer.
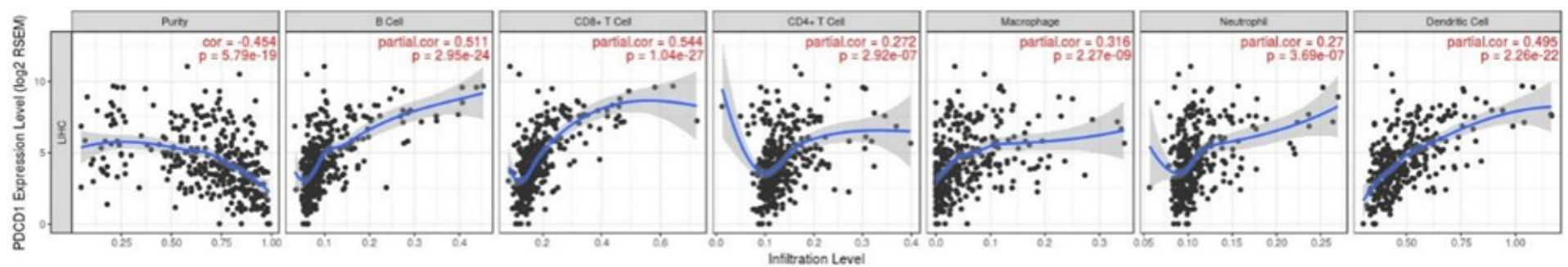

Figure 4

The correlation between PD1 expression level and the infiltration of different immune cells. The red characters "cor" and " $p$ " refer to the correlation coefficient and statistical difference between PD1 and the degree of immune cell infiltration respectively. 